\title{
Targeting Astroglial Glucose Metabolism to Treat Alzheimer Disease
}

\author{
Helena H. Chowdhury ${ }^{1,2}$ and Robert Zorec ${ }^{1,2 *}$ \\ ${ }^{1}$ Laboratory of Neuroendocrinology - Molecular Cell Physiology, Institute of Pathophysiology, University of Ljubljana, Faculty of Medicine, Slovenia \\ ${ }^{2}$ Laboratory of Cell Engineering, Celica Biomedical, Ljubljana, Slovenia
}

*Corresponding author: Robert Zorec.

Received Date: January 13, 2020

Published Date: January 30, 2020

\section{Introduction}

Alzheimer disease (AD) is a common neurodegenerative disease, contributing to $60-70 \%$ to all cases of dementia, which, according to the World health organization, currently represents 50 million people worldwide. AD manifests in the deterioration of cognitive functions, representing a burden for the patients, families and the society as a whole, yet there are no effective treatments. This likely arises from the lack of understanding the primary mechanisms that lead to the demise of neurons in AD. It is likely that neurodegeneration is secondary to the impairment of key homeostatic mechanisms, which are carried out by neuroglia. Here we highlight the role of astrocytes, abundant neuroglial cells in the central nervous system, providing metabolic support to neuronal networks in the form of aerobic glycolysis. Future therapies should target glycolysis regulation, as this process is impaired in AD.

\section{Alzheimer disease (AD), noradrenergic system and astrocytes}

Currently there is no effective treatment for $\mathrm{AD}$, arguably the most common form of neurodegeneration, which leads to cognitive impairment. At tissue level in the central nervous system (CNS), the hallmarks of $\mathrm{AD}$ are extracellular deposits of fibrillar $\beta$-amyloid ( $A \beta$, senile or neuritic plaques) and intraneuronal accumulation of neurofibrillary tangles consisting of Tau protein aggregates [1]. These are thought to elicit a slow gradual neuron loss decade(s) before the onset of the earliest clinical symptoms [2]. In brief, current view posits that neurodegeneration in $\mathrm{AD}$ reflects neuronspecific deficits.

However, the primary reason for the initiation of neurodegeneration may well be due to a failure in neuroglial cells, which maintain CNS homoeostasis [3]. The role of neuroglia in pathological processes was first noted by Alois Alzheimer himself, who found that glia populated senile plaques were in close contact with damaged neurons [4,5]. In humans, post-mortem AD tissue exhibits astroglial hypertrophy with overexpressed levels of glial fibrillary acidic protein (GFAP), a state termed reactive gliosis [6], which may represent a protective, but sometimes even a neurotoxic cell entity in the evolution of disease [7].

There are several hypotheses explaining the mechanism of $\mathrm{AD}$ development, including mutations in amyloid precursor protein or upstream sorting proteins, impairment of neuron functioning (cholinergic, noradrenergic (Locus coeruleus, LC), or glutamatergic), neuroinflamation and energy handling [8]. However, the link between astroglial failure and AD is less clear. An interesting perspective arises from the concept of "cognitive reserve", defined as the degree of functional impairment in response to neuropathologic events, i.e. a similar extent of brain damage may in different subjects result in different cognitive outcomes $[9,10]$. It has two components: the neural reserve, represented by neural networks opposing pathology, and neural compensation, which reflects in the defensive reaction mounted in response to pathology. Both components are regulated by noradrenergic innervation and involve astroglia. The "neural reserve" has been tested in a study involving 165 patients, monitored annually by 19 cognitive tests [11]. Post-mortem neuropathologic examination revealed that the reduced neuronal density in LC, but not in other brainstem nuclei, was robustly associated with the loss of cognitive function, demonstrating that neural reserve is represented by noradrenergic innervation. Moreover, the loss of LC neurons is very much linked to reactive astrogliosis, which is regulated by noradrenaline (NA) [12]. 


\section{Aerobic glycolysis in the central nervous system resides in astrocytes}

In normal ageing LC neurons are reduced in number up to $25 \%$ of those neurons, responsible for $\sim 70 \%$ of brain NA levels, are being lost in the elderly ( $>90$ years age) [13]. Several possibilities exist to reverse the consequences of deficient noradrenergic transmission during neurodegeneration. One of these is linked to a dysfunction in glucose metabolism, also observed clinically [14]. Glucose is considered the primary source of energy in the brain, a vast energy consuming organ, consuming over one fifth of blood glucose at rest [15]. Glucose entry into the CNS from the systemic circulation takes place mainly through astrocytes [16], with their end-feet in contact with blood vessels [17]. Moreover, astrocytes are the only cell type in the brain able to synthesize glycogen, an energy reserve [18]. During the periods of increased energy demands, elicited during arousal and attention through the LC neurons, leading to enhanced neuronal activity, or under hypoglycemic conditions when normal glucose availability is insufficient for neuronal activity, stored glycogen is degraded and metabolized to L-lactate [19]. These divergent glucose pathways are part of the glycogen shunt [20]. Thus astrocytes are essential as a perpetual energy buffer for neurons and an important source of L-lactate [21], which may be transferred to fuel energy demanding neurons which lack energy reserve [22]. This transfer is known as the astrocyte-neuron lactate shuttle (ANLS) hypothesis [23] and suggests that astrocytes provide fuel to neurons.

Although both, astrocyte and neurons, metabolize glucose, evidence suggest that in astrocytes the prevailing mechanism of glucose utilization is aerobic glycolysis, also known as the Warburg effect, a characteristic of morphologically plastic and dividing cells [24]. The upregulation of aerobic glycolysis, a hallmark of the frontal cortex [25], likely involves the activation of LC neurons and release of NA [26], by activating ARs on the astroglial plasma membrane. In turn, astrocytes release L-lactate, which acts not only as energy substrate, but also as a signal and triggers elevated firing, i.e. discharge of action potentials in LC neurons [27]. Such communication may likely exist also between LC axon termini and nearby astroglia, since transcranial direct current stimulation also evokes NA-dependent activation of astroglia [28]. When the levels of NA are reduced, the NA-mediated astroglial excitation [10] which includes L-lactate induced cAMP-signaling and autocrine production of L-lactate via a yet unidentified L-lactate receptor, is reduced [29]. Decreased astrocytic aerobic glycolysis can thus lead to AD-related impairments [30] and severity of AD was recently correlated to levels of decreased rates of glycolysis [31]. Astrogliabased strategies to slow-down neurodegeneration were discussed recently and include (i) exposing the subjects and animals to enriched sensory environment or to electrical stimulation; (ii) transplanting noradrenergic neurons/ into the brain; and (iii) applying drugs that elevate NA and or act in a similar manner as agonists of ARs on astroglia, although via different receptors [10]. The latter approach targets aerobic glycolysis.

\section{Targeting astroglial aerobic glycolysis in AD}

In astrocytes the level of glycogen is modulated; adenosine, noradrenaline and serotonin induce glycogenolysis [21], while insulin and the insulin-like growth factor (IGF1), increase the glycogen content [32-34]. Insulin and IGF1 decrease cytosolic levels of free glucose in astrocytes [34], consistent with the insulin-induced glycogen build-up. Whether insulin increases glucose uptake in astrocytes is controversial. It was reported that insulin regulates glucose uptake by activating the astrocytic glucose transporters $[35,36]$, while insulin might suppress the endogenous glucose production - similarly as in hepatocytes [37]; by the inhibition of glucose-6-phosphatase (G6Pase), present in astrocytes [38,39], which inhibits glucose uptake. Astrocytic G6Pase ensures the removal of glucose-6-phosphate (G6P) from the cytosol, thereby preventing the inhibition of hexokinase (HK) and enabling further glucose uptake [39].

Reduced levels of insulin and insulin-like growth factor 1 (IGF1) have been reported in AD brains [40] and authors proposed at the time the term "Type 3 Diabetes", reflecting this as a pathogenic mechanism of neurodegeneration. Interestingly, in glycogen synthase knockout mice it was shown that glycogen is necessary also for associative learning [41].

The results of the Baltimore Longitudinal Study of Aging revealed that the severity of both senile plaques and neurofibrillary pathology is correlated to lower rates of glycolysis and to higher concentrations of brain tissue glucose [31]. Impaired glycolysis was characterized by the expression of key glycolytic enzymes, HK, phosphofructokinase and pyruvate kinase, which were gradually reduced from healthy to symptomatically ill AD patients.

Impaired brain insulin signaling plays a critical role in the loss of memory functions associated with AD [42]. A doubleblind placebo-controlled study on 25 patients revealed, that intranasal insulin application improved retainment of verbal information after a delay, as well as attentiveness and functional status of patients with AD [43]. Moreover, four months of treatment with intranasal insulin improves memory [44], indicating that insulin signaling affects cognition, likely via astrocytes.

\section{Conclusion}

Normal brain function largely depends on astrocyte aerobic glycolysis, and even a small impairment may cause a significant decline of brain activities. Moreover, the energy metabolism of reactive astrocytes may be a determinant of pathologic process that evolves over decades during neurodegeneration. Therefore, pharmacological manipulations of astrocyte aerobic glycolysis may be considered as a target to remedy cognitive decline during neurodegeneration and AD.

\section{Acknowledgement}

This work was supported by grants P3 310, J3 6790, J3 6789, and J3 9266 from the Slovenian Research Agency, by CipKeBip, 
COST Action BM1002, EU COST Action CM1207-GLISTEN, and EU COST Action CM1207 - EuroCellNet.

\section{Conflict of Interest}

No conflict of interest.

\section{References}

1. Selkoe DJ (2001) Alzheimer's disease: genes, proteins, and therapy. Physiol Rev 81: 741-766.

2. Jack CR, Knopman DS, Jagust WJ, Shaw LM, Aisen PS, et al. (2010) Hypothetical model of dynamic biomarkers of the Alzheimer's pathological cascade. Lancet Neurol 9: 119-128.

3. Verkhratsky A, Nedergaard M (2018) Physiology of Astroglia. Physiol Rev 98: 239-389.

4. Alzheimer A (1910) Contributions to the knowledge of the pathological neuroglia and their relationships to the degradation processes in nerve tissue. In: Franz Nissl AA (Ed.), Histological and histopathological work on the cerebral cortex with special attention to the pathological anatomy of mental illnesses Jena: G. Fischer. P. 401-562.

5. Strassnig M, Ganguli M (2005) About a peculiar disease of the cerebral cortex: Alzheimer's original case revisited. Psychiatry (Edgmont) 2: 3033.

6. Pekny M, Pekna M (2016) Reactive gliosis in the pathogenesis of CNS diseases. Biochim Biophys Acta 1862: 483-491.

7. Liddelow SA, Guttenplan KA, Clarke LE, Bennett FC, Bohlen CJ, et al. (2017) Neurotoxic reactive astrocytes are induced by activated microglia. Nature 541: 481-487.

8. Zulfiqar S, Garg P, Nieweg K (2019) Contribution of astrocytes to metabolic dysfunction in the Alzheimer's disease brain. Biol Chem 400: 1113-1127.

9. Stern Y (2009) Cognitive reserve. Neuropsychologia 47: 2015-2028.

10. Zorec R, Parpura V, Verkhratsky A (2018) Preventing neurodegeneration by adrenergic astroglial excitation. FEBS J 285: 3645-3656.

11. Wilson RS, Nag S, Boyle PA, Hizel LP, Yu L, et al. (2013) Neural reserve, neuronal density in the locus ceruleus, and cognitive decline. Neurology 80: $1202-1208$

12. Sofroniew MV (2014) Astrogliosis. Cold Spring Harb Perspect Biol 7: a020420.

13. Marien MR, Colpaert FC, Rosenquist AC (2004) Noradrenergic mechanisms in neurodegenerative diseases: a theory. Brain Res Brain Res Rev 45: 38-78.

14. Rodriguez-Vieitez E, Saint-Aubert L, Carter SF, Almkvist O, Farid K, et al. (2016) Diverging longitudinal changes in astrocytosis and amyloid PET in autosomal dominant Alzheimer's disease. Brain 139(Pt 3): 922-936.

15. Harris JJ, Jolivet R, Attwell D (2012) Synaptic energy use and supply. Neuron 75: 762-777.

16. Tsacopoulos M, Magistretti PJ (1996) Metabolic coupling between glia and neurons. J Neurosci 16: 877-885.

17. Simard M, Arcuino G, Takano T, Liu QS, Nedergaard M (2003) Signaling at the gliovascular interface. J Neurosci 23: 9254-62.

18. Walls AB, Heimburger CM, Bouman SD, Schousboe A, Waagepetersen HS (2009) Robust glycogen shunt activity in astrocytes: Effects of glutamatergic and adrenergic agents. Neuroscience 158: 284-292.

19. Brown AM, Ransom BR (2007) Astrocyte glycogen and brain energy metabolism. Glia 55: 1263-1271.

20. Shulman RG, Hyder F, Rothman DL (2001) Cerebral energetics and the glycogen shunt: neurochemical basis of functional imaging. Proc Natl Acad Sci U S A 98: 6417-6422.

21. Dringen R, Hamprecht B (1993) Differences in glycogen metabolism in astroglia-rich primary cultures and sorbitol-selected astroglial cultures derived from mouse brain. Glia 8: 143-149.

22. Barros LF (2013) Metabolic signaling by lactate in the brain. Trends Neurosci 36: 396-404.

23. Pellerin L, Magistretti PJ (1994) Glutamate uptake into astrocytes stimulates aerobic glycolysis: a mechanism coupling neuronal activity to glucose utilization. Proc Natl Acad Sci USA 91: 10625-10629.

24. Vander Heiden MG, Cantley LC, Thompson CB (2009) Understanding the Warburg effect: the metabolic requirements of cell proliferation. Science 324: 1029-1033.

25. Goyal MS, Hawrylycz M, Miller JA, Snyder AZ, Raichle ME (2014) Aerobic glycolysis in the human brain is associated with development and neotenous gene expression. Cell Metab 19: 49-57.

26. Dienel GA, Cruz NF (2016) Aerobic glycolysis during brain activation: Adrenergic regulation and influence of norepinephrine on astrocytic metabolism. J Neurochem 138(1): 14-52.

27. Tang F, Lane S, Korsak A, Paton JF, Gourine AV, et al. (2014) Lactatemediated glia-neuronal signalling in the mammalian brain. Nat Commun 5: 3284.

28. Hiromu Monai, Masamichi Ohkura, Mika Tanaka, Yuki Oe, Ayumu Konno, et al. (2016) Calcium imaging reveals glial involvement in transcranial direct current stimulation-induced plasticity in mouse brain. Nat Commun 7: 11100 .

29. Vardjan N, Chowdhury HH, Horvat A, Velebit J, Malnar M, et al. (2018) Enhancement of Astroglial Aerobic Glycolysis by Extracellular LactateMediated Increase in cAMP. Frontiers in Molecular Neuroscience 11: 148.

30. Yao J, Rettberg JR, Klosinski LP, Cadenas E, Brinton RD (2011) Shift in brain metabolism in late onset Alzheimer's disease: implications for biomarkers and therapeutic interventions. Mol Aspects Med 32: 247257.

31. An Y, Varma VR, Varma S, Casanova R, Dammer E, et al. (2018) Evidence for brain glucose dysregulation in Alzheimer's disease. Alzheimers Dement 14: 318-329.

32. Dringen R, Hamprecht B (1992) Glucose, insulin, and insulin-like growth factor I regulate the glycogen content of astroglia-rich primary cultures. J Neurochem 58: 511-517.

33. Kum W, Zhu SQ, Ho SK, Young JD, Cockram CS (1992) Effect of insulin on glucose and glycogen metabolism and leucine incorporation into protein in cultured mouse astrocytes. Glia 6: 264-268.

34. Muhic M, Vardjan N, Chowdhury HH, Zorec R, Kreft M (2015) Insulin and Insulin-like Growth Factor 1 (IGF-1) Modulate Cytoplasmic Glucose and Glycogen Levels but Not Glucose Transport across the Membrane in Astrocytes. J Biol Chem 290: 11167-11176.

35. Garcia-Caceres C, Quarta C, Varela L, Gao Y, Gruber T, et al. (2016) Astrocytic Insulin Signaling Couples Brain Glucose Uptake with Nutrient Availability. Cell 166: 867-880.

36. Hamai M, Minokoshi Y, Shimazu T (1999) L-Glutamate and insulin enhance glycogen synthesis in cultured astrocytes from the rat brain through different intracellular mechanisms. J Neurochem 73: 400-407.

37. van Schaftingen E, Gerin I (2002) The glucose-6-phosphatase system. Biochem J 362: 513-532.

38. Csala M, Marcolongo P, Lizak B, Senesi S, Margittai E, et al. (2007) Transport and transporters in the endoplasmic reticulum. Biochim Biophys Acta 1768: 1325-1341.

39. Muller MS, Fouyssac M, Taylor CW (2018) Effective Glucose Uptake by Human Astrocytes Requires Its Sequestration in the Endoplasmic Reticulum by Glucose-6-Phosphatase-beta. Curr Biol 28: 3481-3486.e4.

40. Steen E, Terry BM, Rivera EJ, Cannon JL, Neely TR, et al. (2005) Impaired insulin and insulin-like growth factor expression and signaling mechanisms in Alzheimer's disease--is this type 3 diabetes? J Alzheimers Dis 7: 63-80. 
41. Duran J, Saez I, Gruart A, Guinovart IJ, Delgado-Garcia JM (2013) Impairment in long-term memory formation and learning-dependent synaptic plasticity in mice lacking glycogen synthase in the brain. J Cereb Blood Flow Metab 33: 550-556.

42. Benedict C, Frey WH, Schioth HB, Schultes B, Born J, et al. (2011) Intranasal insulin as a therapeutic option in the treatment of cognitive impairments. Exp Gerontol 46: 112-115.
43. Reger MA, Watson GS, Green PS, Wilkinson CW, Baker LD, et al. (2008) Intranasal insulin improves cognition and modulates beta-amyloid in early AD. Neurology 70: 440-448

44. Craft S, Claxton A, Baker LD, Hanson AJ, Cholerton B (2017) Effects of Regular and Long-Acting Insulin on Cognition and Alzheimer's Disease Biomarkers: A Pilot Clinical Trial. J Alzheimers Dis 57: 1325-1334. 